\title{
Immobilization of Cell-Adhesive Laminin Peptides in Degradable PEGDA Hydrogels Influences Endothelial Cell Tubulogenesis
}

\author{
Saniya Ali,, Jennifer E. Saik, Dan J. Gould,, ${ }^{1,3}$ Mary E. Dickinson, ${ }^{3}$ and Jennifer L. West ${ }^{1,2}$
}

\begin{abstract}
Attachment, spreading, and organization of endothelial cells into tubule networks are mediated by interactions between cells in the extracellular microenvironment. Laminins are key extracellular matrix components and regulators of cell adhesion, migration, and proliferation. In this study, laminin-derived peptides were conjugated to poly(ethylene glycol) (PEG) monoacrylate and covalently incorporated into degradable PEG diacrylate (PEGDA) hydrogels to investigate the influence of these peptides on endothelial cellular adhesion and function in organizing into tubule networks. Degradable PEGDA hydrogels were synthesized by incorporating a matrix metalloproteinase (MMP)-sensitive peptide, GGGPQGIWGQGK (abbreviated PQ), into the polymer backbone. The secretion of MMP-2 and MMP-9 by endothelial cells promotes polymer degradation and consequently cell migration. We demonstrate the formation of extensive networks of tubule-like structures by encapsulated human umbilical vein endothelial cells in hydrogels with immobilized synthetic peptides. The resulting structures were stabilized by pericyte precursor cells $(10 \mathrm{~T} 1 / 2 \mathrm{~s})$ in vitro. During tubule formation and stabilization, extracellular matrix proteins such as collagen IV and laminin were deposited. Tubules formed in the matrix of metalloproteinase sensitive hydrogels were visualized from 7 days to 4 weeks in response to different combination of peptides. Moreover, hydrogels functionalized with laminin peptides and transplanted in a mouse cornea supported the ingrowth and attachment of endothelial cells to the hydrogel during angiogenesis. Results of this study illustrate the use of laminin-derived peptides as potential candidates for modification of biomaterials to support angiogenesis.
\end{abstract}

Key words: angiogenesis; biomaterials; extracellular matrix; peptides; tissue engineering

\section{Introduction}

S UCCESSFUL THERAPIES IN REGENERATIVE MEDICINE have $\checkmark$ been primarily limited to thin or avascular tissues, such as skin, cartilage, and bladder in which oxygen, nutrients, and wastes diffuse through the extracellular space unassisted. ${ }^{1,2}$ Generating larger and more complex tissues, such as the heart, kidneys, or liver, requires the formation of a stable microvascular network for prolonged cell viability and function. The normal development of blood vessels in the body is mediated by signals between endothelial cells and the extracellular matrix (ECM). These matrix interactions play an important part in many endothelial cell functions, including adhesion, proliferation, migration, and differentiation., Based on our knowledge of this interplay, synthetic scaffolds can be designed to mimic the vascular endothelial cell micro- environment in an effort to guide and support blood vessel formation in engineered tissues.

The vascular lumen is a hollow space composed of an endothelial cell layer and an external sheath of mural cells separated by a laminin-rich basement membrane. Laminins, which are critical to the structure of these tissues, make up a family of heterotrimeric glycoproteins, with each protein containing one $\alpha, \beta$, and $\gamma$ chain., 5 The $\alpha, \beta$, and $\gamma$ subunits characterize the functions and properties of the laminin proteins. For example, the $\alpha$ chain is central in determining integrin ligand specificity, whereas, the $\beta$ subunit connects to the cytoskeleton and affects multiple signaling pathways. ${ }^{7} \mathrm{Sev}-$ eral cell binding motifs based on laminin-derived peptides have been investigated for approaches to modulate cell adhesion and spreading, including those based on the RGD, IKVAV, and YIGSR amino acid sequences. ${ }^{8,9}$ The extensively

\footnotetext{
${ }^{1}$ Department of Bioengineering, Rice University, Houston, Texas.

${ }^{2}$ Department of Biomedical Engineering, Duke University, Durham, North Carolina.

${ }^{3}$ Department of Molecular Physiology and Biophysics, Baylor College of Medicine, Houston, Texas.
} 
studied RGD sequence is found in many ECM proteins and serves as the binding motif for cell surface integrin receptors. For example, human umbilical vein endothelial cells (HUVECs) seeded upon RGDS-modified poly(ethylene glycol) diacrylate (PEGDA) hydrogels showed increased spreading and adhesion 24-h postseeding compared with the same cells on the unmodified polymer or polymer modified with RGES. ${ }^{10}$ In other work with this peptide, poly(ethylene glycol) (PEG) hydrogels functionalized with protease-cleavable peptide sequences and RGDS showed that immobilized RGDS facilitated initial cell adhesion, while degradation of the polymer from cell-secreted matrix metalloproteinases (MMPs) promoted the formation of vascular networks in cocultures of endothelial and mesenchymal stem cell progenitor cells. ${ }^{11}$ Furthermore, adhesion and proliferation studies using HUVECs indicated that cells spread better on the RGDSmodified surface. ${ }^{12,13}$

IKVAV, a peptide derived from the $\alpha$-chain of laminin, has been previously shown to induce endothelial cell adhesion and subsequent tubule formation. In a study conducted by Grant et al.,' ${ }^{14}$ the IKVAV peptide demonstrated increased endothelial cell mobilization, capillary branching, and vessel formation in both a murine angiogenesis assay and experiments with the chick yolk sac/chorioallantoic membrane. In the presence of immobilized IKVAV on collagen type I hydrogels, vascular endothelial cells stimulated cell migration, adhesion, and capillary network formation. ${ }^{15}$ This peptide has also been shown to mediate revascularization of ischemic tissue, ${ }^{16}$ making it of great interest to those seeking to induce microvascular networks de novo.

The integrin receptor ligand YIGSR, found in a cysteinerich site of the laminin $\beta$ chain, has been extensively investigated as a mediator of cell adhesion. ${ }^{17,18}$ Various studies have demonstrated that YIGSR plays a key role in endothelial cell-to-cell interactions and tubule formation. ${ }^{12,19,20}$ In one study, incorporation of YIGSR in a polyurethane urea scaffold resulted in improvement in endothelial cell adhesion, proliferation, migration, and ECM production. ${ }^{18-21}$ In another study, surface presentation of both RGD and YIGSR peptides was shown to induce endothelial cell tubule formation over unmodified materials. ${ }^{19}$ When RGD and YIGSR were tested separately, YIGSR-modified matrigel induced endothelial cells to organize in tubules, while RGD-modified matrigel only supported cell attachment, providing key evidence that these peptides have separate effects on endothelial cells with the primary role of RGD in cell attachment and YIGSR in tubule formation. ${ }^{19}$

In the present study, we demonstrate that the degree of endothelial cell tubule formation varies with the peptide sequence presented in the material. PEGDA hydrogels were selected as the synthetic scaffold for these studies due to their innate ability to resist protein adsorption and nonspecific cell adhesion. ${ }^{11}$ This polymeric material acts as a "blank slate" into which selective functionality can be incorporated by covalent attachment of proteins and peptides, including those that mediate cell adhesion and others that are susceptible to biodegradation. As such, this creates a controlled microenvironment in which interactions with cells are driven primarily by the incorporated biomolecules. Importantly, PEGDA hydrogels are generated in a photocrosslinking process consisting of mild conditions that permit cellular encapsulation with high viability. ${ }^{11,22}$
Laminin-derived peptides were conjugated to heterobifunctional PEG chains and covalently incorporated in hydrogels. In response to these immobilized peptides, encapsulated HUVECs organized into extensive networks of capillary-like three-dimensional (3D) structures. Pericyte precursor cells $(10 \mathrm{~T} 1 / 2 \mathrm{~s})$ stabilized the resulting structures. Additionally, hydrogels incorporated with laminin-derived peptides and implanted in the mouse cornea stimulated the formation of functional blood vessels. This work shows that YIGSR, IKVAV, and RGD peptides have different abilities to modulate vessel assembly and long-term tubule stability both in vitro and in vivo. Based on these results, we believe that laminin-derived peptides can be immobilized in synthetic scaffolds and exploited as a strategy for regulating the formation of microvasculature in tissue-engineered constructs.

\section{Material and Methods}

\section{Cell maintenance}

HUVECs (Lonza, Walkersville, MD) were grown in endothelial growth medium (EGM-2), supplemented with ascorbic acid, epidermal growth factor, fibroblast growth factor (hFGF-2), heparin, hydrocortisone, insulin-like growth factor, GA-1000 (gentamicin, amphotericin-B), and 2\% fetal bovine serum (Lonza). 10T1/2 pericyte precursor cells (American Type Culture Collection, Manassas, VA) were cultured in Dulbecco's modified Eagle's medium (DMEM; Gibco, North Andover, MA) supplemented with $10 \%$ fetal bovine serum and $2 \mathrm{mM}$ L-glutamine, $1000 \mathrm{U} / \mathrm{mL}$ penicillin, and $100 \mathrm{mg}$ / L streptomycin (Sigma, St. Louis, MO). Experiments were conducted with HUVECs from passages 2 to 5, and 10T1/2 cells from passages 15 to 18 . All cells were incubated at $37^{\circ} \mathrm{C}$ and at $5 \% \mathrm{CO}_{2}$.

\section{Peptide synthesis}

The laminin-derived sequences YIGSR and IKVAV were synthesized on an APEX 396 solid phase peptide synthesizer (AAPPTec, Louisville, KY) using standard Fmoc chemistry. After purification, the peptides were characterized via matrix-assisted laser desorption/ionization-time-of-flight mass spectrometry (MALDI-ToF; Bruker Daltonics, Billerica, MA). The same methods were used to generate the proteasesensitive peptide sequence, GGGPQGIWGQGK (PQ), which has been shown to render synthetic hydrogels degradable when incorporated into the polymer backbone. ${ }^{11,22} \mathrm{MMP}-2$ and MMP-9, secreted by endothelial cells, cleave the PQ peptide in the polymer backbone, resulting in the degradation of PEG hydrogel.

\section{Polymer conjugation}

The cell-adhesive peptide RGDS (American Peptide, Sunnyvale, CA), was conjugated to acryloyl-PEG-succinimidyl carboxymethyl (PEG-SCM, 3400 Da, Laysan Bio Inc., Arab, AL) to form PEG-RGDS (Fig. 1). In this reaction, the RGDS peptide was first dissolved in anhydrous dimethyl sulfoxide with $2 \mu \mathrm{L}$ of diisopropylethylamine and then added drop wise to dry acryloyl-PEG-succinimidyl carboxymethyl at a molar ratio of 1:1.2 (PEG:peptide). The resulting product was then purified via dialysis against water in a regenerated cellulose membrane (MWCO 5000; Spectrum Laboratories, Inc., Rancho Dominguez, CA) and lyophilized. Successful 


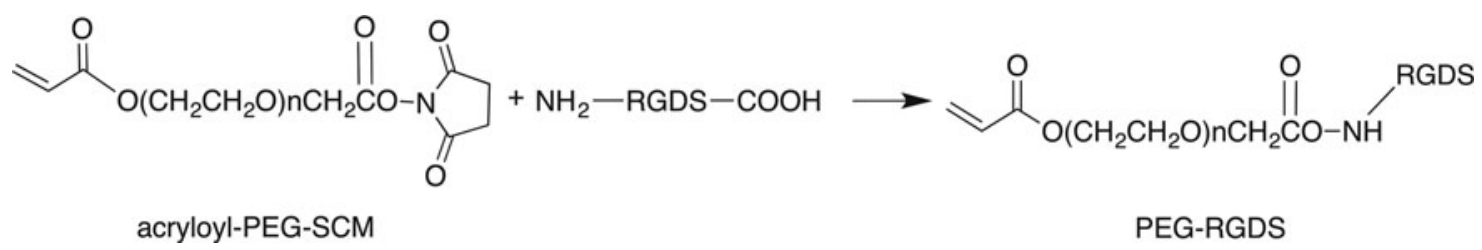

FIG. 1. The cell-adhesive peptide, RGDS, was conjugated to acryloyl-PEG-SCM (3400 Da) to form PEG-RGDS. Similar reactions were used to conjugate the additional laminin peptides, yielding PEG-IKVAV and PEG-YIGSR. PEG, poly(ethylene glycol); SCM, succinimidyl carboxymethyl.

conjugation of the peptide to PEG was confirmed via a gel permeation chromatography system equipped with UV-VIS and evaporative light scattering detectors (GPC, Polymer Laboratories, Amherst, MA).. The same protocols were followed for the laminin peptides, YIGSR and IKVAV, to yield PEG-IKVAV and PEG-YIGSR. The PQ peptide conjugation was achieved in process similar to that for PEG-RGDS but with a PEG-SCM:PQ peptide molar ratio of 2:1 generating an MMP-degradable segment that was flanked on both sides by PEG (PEG-PQ-PEG).

\section{Encapsulation of endothelial cells and 10T1/2 pericyte precursors in degradable hydrogels}

HUVECs and 10T1/2 cells were encapsulated in degradable hydrogels in order to observe the effects of covalently immobilized IKVAV and YIGSR on tubule formation. HUVECs and 10T1/2 cells were encapsulated at ratio of 4:1. Hydrogels were formed from prepolymer solutions composed of 10\% (w/v) PEG-PQ-PEG in sterile HEPES buffered saline (HBS) containing $1.5 \%(\mathrm{v} / \mathrm{v})$ triethanolamine, $1 \mathrm{mM}$ $\operatorname{eosin} \mathrm{Y}$, and $3.95 \mu \mathrm{L} / \mathrm{mL}$ N-vinyl-2-pyrrolidone (NVP). PEG-peptides were added to the prepolymer solution to create four treatment groups: (1) $3.5 \mathrm{mM}$ PEG-IKVAV, (2) $3.5 \mathrm{mM}$ PEG-YIGSR, (3) $3.5 \mathrm{mM}$ PEG-IKVAV with $1.0 \mathrm{mM}$ PEG-RGDS, and (4) $3.5 \mathrm{mM}$ PEG-YIGSR with $1.0 \mathrm{mM}$ PEGRGDS. A prepolymer solution containing $3.5 \mathrm{mM}$ PEGRGDS alone was used as a control group. HUVECs and 10T1/2 cells were harvested using trypsin-EDTA and pelleted by centrifuging at $700 \mathrm{~g}$ for $4 \mathrm{~min}$. The cell pellets were then resuspended in the prepolymer solutions to concentrations of 10,000 cells $/ \mu \mathrm{L}$. Five-microliter droplets of cell-laden polymer were sandwiched between a Sigmacoated (Sigma) glass slide and an acrylated cover glass separated by a $380-\mu \mathrm{m}$ polydimethylsiloxane (PDMS) spacer. The solutions were cross-linked under white light for $35 \mathrm{sec}$ to form hydrogels that were then immersed in EGM-2 media and incubated at $37^{\circ} \mathrm{C}$ in a $5 \% \mathrm{CO}_{2}$ environment for up to 28 days. Media on the hydrogels was replenished every other day.

\section{Assessing tubule morphology and ECM protein production}

Endothelial cell tubule formation was evaluated by measuring total tubule length per field of view and average tubule diameter in hydrogels that were stained to visualize actin filaments and cell nuclei. Hydrogels were first fixed in $4 \%$ paraformaldehyde for $20 \mathrm{~min}$ and then washed with phosphate-buffered saline (PBS, pH 7.4). Next, a $0.5 \%$ Triton-X solution was added for $30 \mathrm{~min}$ to permeabilize encapsulated cells. Following another PBS wash, nonspecific binding was blocked by incubation with $1 \%$ bovine serum albumin (BSA) overnight. Gels were finally exposed to a 1:100 dilution of rhodamine phalloidin (Invitrogen, Carlsbad, CA) containing $2 \mu \mathrm{M} \mathrm{4}$ ',6-diamidino-2-phenylindole (DAPI) in HBS with $0.1 \%$ BSA for $2 \mathrm{~h}$ to stain actin and cell nuclei, respectively. The hydrogels were visualized using a confocal microscope (Zeiss 5-LIVE, Plan-Apochromat $20 \times$ objective with $0.8 \mathrm{NA}$ ) with a z-stack depth of $20 \mu \mathrm{m}$. Imaris analysis software (Bitplane Inc., South Windsor, CT) was used to trace and measure tubule length and tubule diameter in three fields of view for each hydrogel $(n=3$ hydrogels per experimental group, $\mathrm{FOV}=318 \mu \mathrm{m} \times 318 \mu \mathrm{m}){ }^{23,24}$

To elucidate collagen IV and laminin production, hydrogels were first blocked with $1 \%$ BSA overnight and then incubated with primary antibodies on a rocker table at $4^{\circ} \mathrm{C}$ for an additional overnight period to insure antibody infiltration into the samples. For these studies, primary antibodies included rabbit anticollagen IV (1:100 in PBS with 0.1\% BSA, Abcam, Cambridge, MA) and chicken antilaminin (1:200 in PBS with $0.1 \%$ BSA, Sigma). Hydrogels were rinsed in PBS five times at 1-h intervals and then incubated with a 1:400 dilution of Alexa Fluor 488 donkey antirabbit IgG (Invitrogen) or Alexa Fluor 488 goat antichicken IgG (Invitrogen) overnight at $4^{\circ} \mathrm{C}$ to visualize the primary antibodies. Subsequently, samples were rinsed with PBS and then imaged using a confocal microscope under a $20 \times$ objective. Image slices of $1-\mu \mathrm{m}$ thickness from a z-stack of $20 \mu \mathrm{m}$ deep in the gel were taken for three fields of view from each of three hydrogels. Relative amounts of laminin and collagen IV were determined by measuring Alexa Fluor 488 pixel intensity in ImageJ (National Institutes of Health, Bethesda, MD) and normalizing to cell number using the DAPI pixel intensity.

\section{In vivo angiogenesis assay: hydrogel implantation into the mouse cornea}

All animal procedures applied in this study were approved by the Institutional Animal Care and Use Committee (IACUC) at Baylor College of Medicine. In order to assess the effects of laminin peptides on tubule formation in vivo, degradable PEGDA hydrogels were implanted into the corneas of Flk1myr::mCherry transgenic mice according to previously published procedures. ${ }^{25}$ Transgenic mice express an endothelial cell-specific fluorescent protein that allows formed tubules to be visualized via fluorescence microscopy. ${ }^{26}$ Briefly, a Von Graefe knife was used to generate micropockets by partial thickness incisions into the cornea of anesthetized mice. Hydrogels were formed from prepolymer solutions composed of $10 \%(\mathrm{w} / \mathrm{v})$ PEG-PQ-PEG in sterile HBS containing $10 \mu \mathrm{L} /$ $\mathrm{mL}$ of $300 \mathrm{mg} / \mathrm{mL}$ 2,2-dimethoxy-2-phenylacetophenone 
(DMAP) in NVP and $320 \mathrm{ng}$ of soluble PDGF-BB and $80 \mathrm{ng}$ of FGF-2 per gel. PDGF-BB and FGF-2 were added to the gels to initiate the angiogenic response of vessels from the limbus of the eye. PEG-peptides were added to the prepolymer solution to create four treatment groups: (1) $3.5 \mathrm{mM}$ PEG-IKVAV, (2) $3.5 \mathrm{mM}$ PEG-YIGSR, (3) $3.5 \mathrm{mM}$ PEG-IKVAV with $1.0 \mathrm{mM}$ PEG-RGDS, and (4) $3.5 \mathrm{mM}$ PEG-YIGSR with $1.0 \mathrm{mM}$ PEGRGDS. A prepolymer solution containing $3.5 \mathrm{mM}$ PEGRGDS alone was used as a control. Once hydrogel solutions were formulated, $0.12 \mu \mathrm{L}$ of polymer solution was injected into a mold made of two glass slides separated by a $125-\mu \mathrm{m}$ PDMS spacer. Following $2 \mathrm{~min}$ of exposure to UV light $\left(365 \mathrm{~nm}, 10 \mathrm{~mW} / \mathrm{cm}^{2}\right)$, the polymerized gels were immediately implanted in the cornea micropockets. For analysis of blood vessel structure and angiogenesis, mice corneas were harvested 7 days postimplantation and fixed in $4 \%$ formaldehyde. A 543-nm laser was used to excite the mCherry fluorophore in order to visualize and acquire z-stack images of endothelial cells that had migrated and invaded into the modified hydrogels. FARSIGHT software (http:// farsight-toolkit.org/wiki/Main_Page) was used to measure vessel diameter and count vessel branch points in the acquired images $(n=5-7)$. The space filling parameters of fractal dimension, lacunarity, and vessel density were also quantified as previously reported ${ }^{27}$ to examine the extent of the angiogenic response to the modified hydrogels $(n=5-7)$. The lacunarity parameter describes the spatial distribution between vessel beds, ${ }^{27}$ and fractal dimension uses box-counting algorithms to measure object morphology, which helps to quantify the complexity of vessel beds. ${ }^{27}$

\section{Statistics}

Single factor ANOVA and subsequent Bonferroni post hoc tests were used to analyze vessel formation and ECM production. For each analysis, $p<0.05$ was considered to be significant.

\section{Results}

\section{Encapsulated endothelial cells exposed to laminin-derived peptides form tubule networks}

HUVECs and pericyte-precursor cells encapsulated in degradable PEGDA hydrogels underwent tubulogenesis with the extent of this response dependent upon the combination of laminin-derived peptides included in the polymer matrix. The tubule networks were visualized as early as 7 days after encapsulation by staining for cell nuclei via DAPI and actin filaments via phalloidin (Fig. 2A). Tubules persisted up to 28 days, with the highest tubule formation response observed in hydrogels with both PEG-YIGSR and PEG-RGDS. To quantify the tubule formation response, average tubule diameter and total tubule length were calculated and evaluated using a single-factor ANOVA and Bonferroni post hoc test (Supplementary Fig. S1). All groups, when compared individually, showed an increase in tubule diameter and total
A

PEG-RGDS

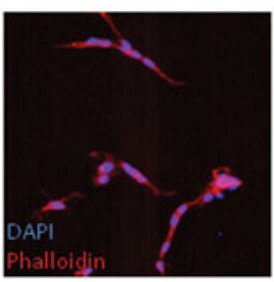

$\mathbf{B}_{25}$

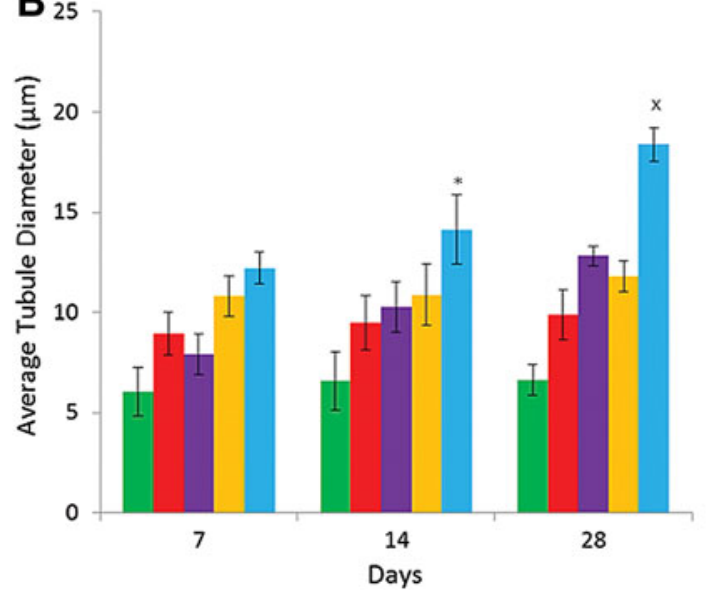

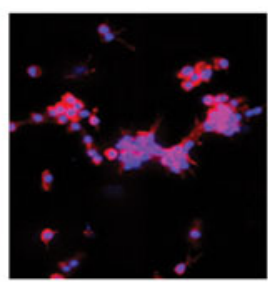

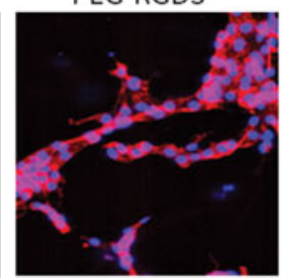

C

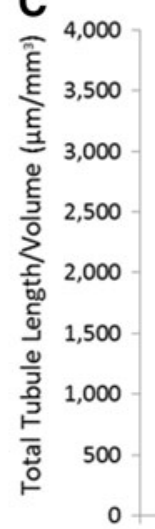

PEG-YIGSR

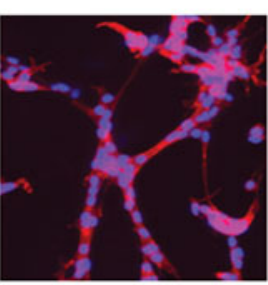

- PEG-RGDS

-PEG-IKVAV

- PEG-IKVAVIPEG-RGDS

=PEG-YIGSR

\#PEG-YIGSR/PEG-RGDS

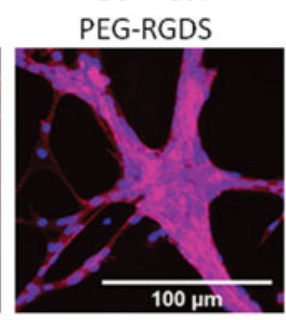

PEG-YIGSR

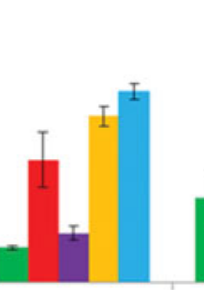

7

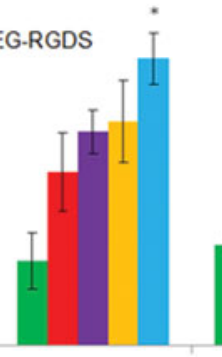

14

Days

FIG. 2. Images of cells in modified hydrogels (A) were taken after 7 days in culture. To quantify the resulting tubule formation in vitro, the average tubule diameter (B) and total tubule length per field of view (C) were calculated. *Significantly different from all other groups for time point 14 days in culture $(p<0.01)$. ${ }^{\mathrm{x}}$ Significantly different from all other groups at time point 28 days in culture $(p<0.01)$. Supplementary Fig. S1 shows all individual significant differences between laminin peptide groups and their effect on average tubule diameter and total tubule length per volume. 
tubule length over time (Fig. 2B, 2C). Additionally, cells that were exposed to a combination of PEG-YIGSR and PEGRGDS had a significantly higher tubule length and tubule diameter when compared with all other treatment groups $(p<0.01)$.

\section{Collagen IV and laminin expression in tubule networks}

ECM proteins are generated by vessel cells in the process of tubule formation and establishment of long-term stability. In light of this, immunohistochemistry was performed to measure production of collagen IV and laminin in the experimental hydrogels. Regions of defined tubule formation in hydrogels were found to correspond to areas with high amounts of collagen IV deposition (as measured by pixel intensity). Specifically, cells exposed to a combination of PEGYIGSR and PEG-RGDS showed the highest expression of this important ECM protein (Fig. 3), which was further found to increase over time and to be dependent on the type peptide sequences presented. In treatment groups with PEG-YIGSR alone and combinations of PEG-IKVAV or PEG-YIGSR with PEG-RGDS, the amount of collagen IV increased significantly from 7 to 28 days $(p<0.05)$ as tubules were stabilized and maturing. For all time points, collagen IV production by cells was significantly higher $(p<0.01)$ in gels with PEG-YIGSR compared with those with PEGIKVAV. Furthermore, the addition of PEG-RGDS to PEGIKVAV or PEG-YIGSR induced more collagen production than PEG-IKVAV or PEG-YIGSR being presented alone. For example, at 14 days, the amount of collagen production in hydrogels with PEG-YIGSR co-immobilized with PEGRGDS was 35\% greater than with PEG-YIGSR alone. Complete Bonferroni post hoc analysis of this data is provided in Supplementary Fig. S2.

Laminin deposition was also highly dependent on the peptide sequences presented, as evidenced by significantly higher $(p<0.05)$ protein accumulation in all treatment groups containing PEG-YIGSR compared with those with PEGIKVAV alone (Fig. 4). Specifically, at 7 days hydrogels with PEG-YIGSR contained 25\% more laminin than those with PEG-IKVAV.

\section{PEG-laminin peptides enhance the in vivo vascular response}

To investigate the impact of laminin-derived peptides in vivo, hydrogels were implanted into corneal micropockets created in Flk1-myr::mCherry transgenic mice. Using this wellestablished angiogenesis assay, ${ }^{11,25}$ we demonstrated that the addition of IKVAV and YIGSR peptides to PEGDA hydrogels enhances cell-cell attachment and organization as visualized by the mCherry fluorescence of mouse endothelial cells (Fig. 5). Soluble PDGF-BB and FGF-2 were added to the hydrogels with immobilized peptides to induce angiogenesis from the limbal vessels of the eye. Covalent immobilization of either PEG-IKVAV or PEG-YIGSR with PEG-RGDS produced tubules with a significantly greater vessel density and tubule branching than did treatment groups with either peptide
A

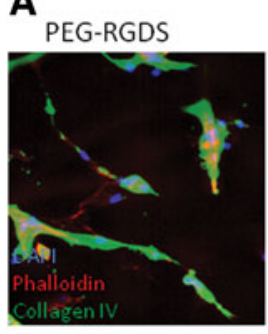

B
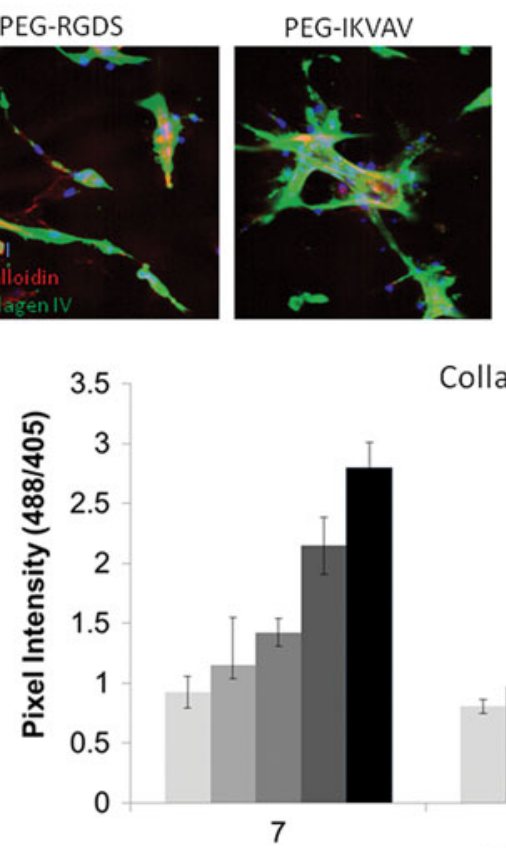

PEG-IKVAV PEG-RGDS

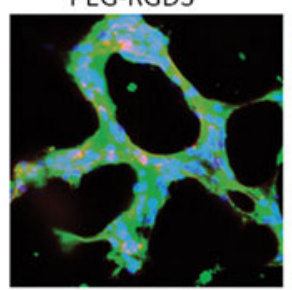

PEG-YIGSR

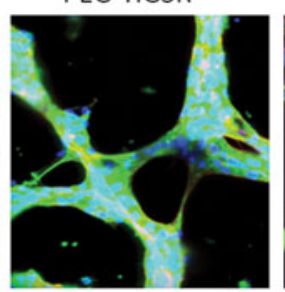

PEG-YIGSR

PEG-RGDS

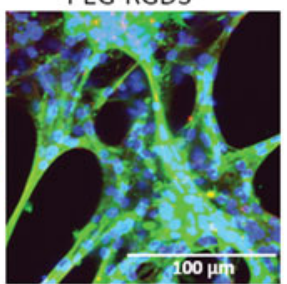

Collagen IV Production

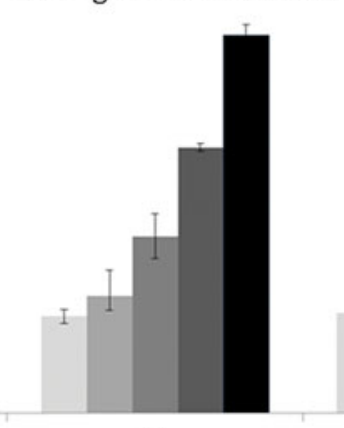

14

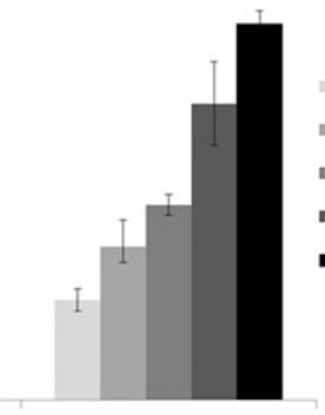

- PEG-RGDS

॥ PEG-IKVAV

॥ PEG-IKVAVIPEG-RGDS

- PEG-YIGSR

- PEG-YIGSR/PEG-RGDS

Time (Days)

FIG. 3. The amount of extracellular matrix (ECM) proteins produced by tubules was dependent on the type of peptide presented to encapsulated cells. Images of cells in modified hydrogels (A) were taken after 14 days in culture (B). Laminin deposition was quantified and was found to correlate to the growth of tubules. Increase in laminin production (shown in green) by cells was observed as the time in culture was prolonged from 7 days to 28 days. Refer to Supplementary Fig. S2 for individual significant differences between laminin peptide groups and their effect on collagen IV production. 
A

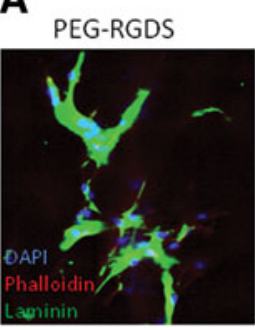

B
PEG-IKVAV PEG-RGDS

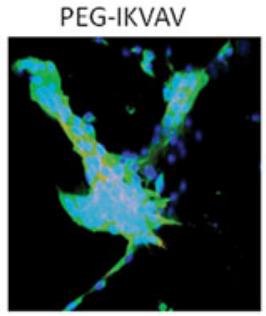

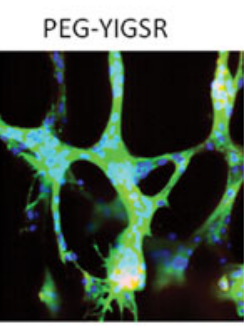

PEG-YIGSR PEG-RGDS

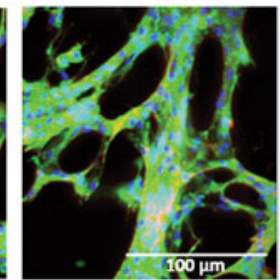

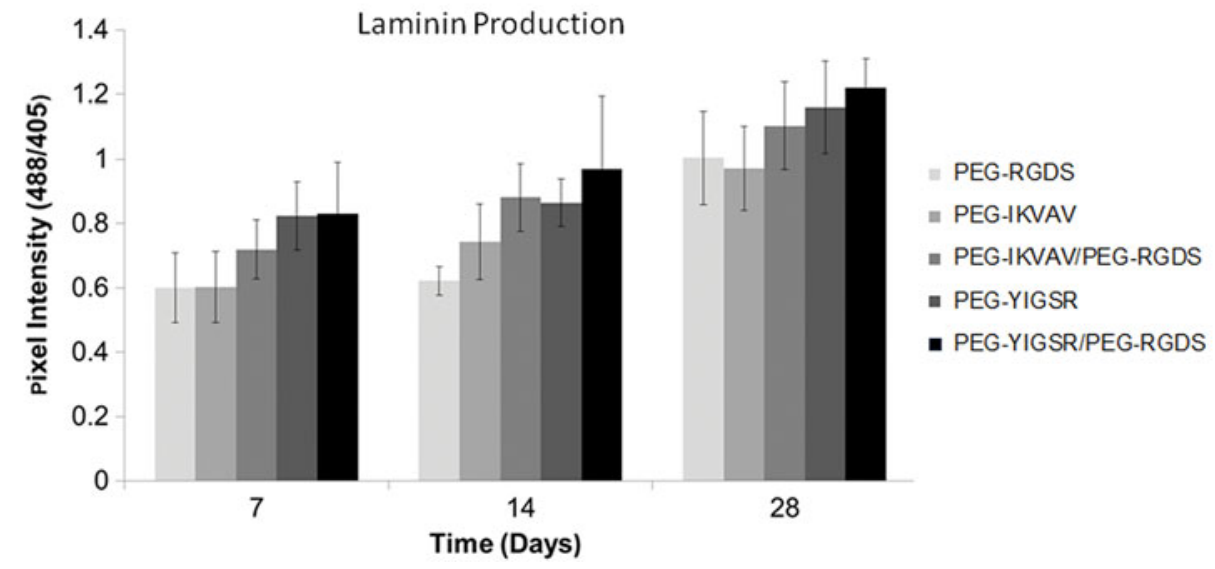

FIG. 4. The amount of ECM proteins produced by tubules was dependent on the type of peptide presented to encapsulated cells. Images of cells in modified hydrogels (A) were taken after 14 days in culture (B). Collagen IV deposition shown in green was quantified and found to correspond to areas of high tubule formation. This result is noticeable for all time points up to 28 days for the PEG-YIGSR/PEG-RGDS combination. Refer to Supplementary Fig. S2 for individual significant differences between laminin peptide groups and their effect on laminin production.

A
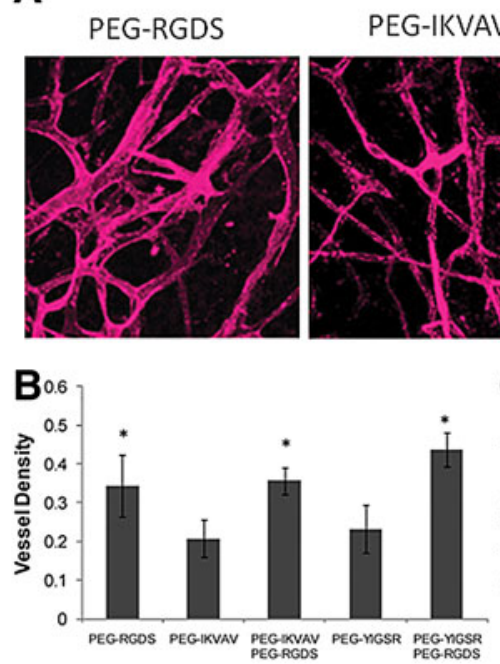

PEG-IKVAV

PEG-RGDS

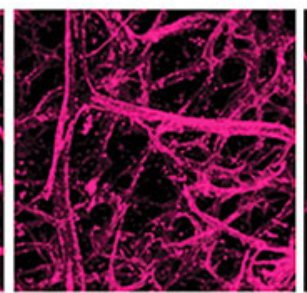

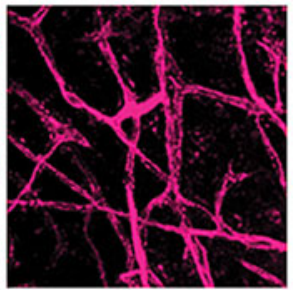

$\mathbf{C}_{1.95}$
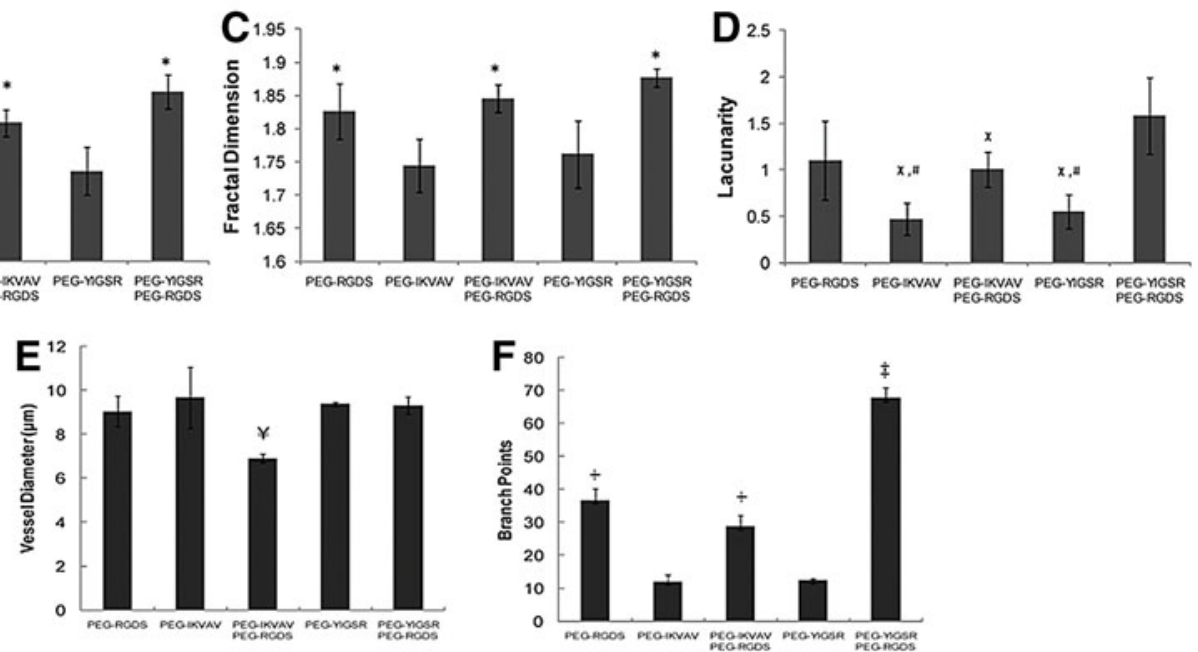

FIG. 5. As visualized by the mCherry fluorescence of mouse endothelial cells (A), the presence of PEG-YIGSR and PEG-RGDS induced a significant difference in vessel density (B), fractal dimension (C), lacunarity (D), vessel diameter (E), and branch points (F). * Significantly different from PEG-YIGSR and PEG-IKVAV $(p<0.01)$. ${ }^{\times}$Significantly different from PEG-YIGSR and PEG-RGDS $(p<0.05)$. " Significantly different from PEG-RGDS $(p<0.05) .{ }^{{ }^{*}}$ Significantly different from all other groups $(p<0.05)$. Scale bar $=50$ $\mu \mathrm{m}$. 'Significantly different from all other groups $(p<0.01)$. ${ }^{+}$Significantly different from PEG-YIGSR and PEG-IKVAV $(p<0.05)$. 
alone $(p<0.01)$. Additionally, space-filling parameters were analyzed to quantify vessel morphology. With the inclusion of both PEG-YIGSR and PEG-RGDS in implanted hydrogels, vessels with a higher lacunarity parameter $(p<0.05)$ and greater fractal dimension $(p<0.01)$ were produced, indicative of more complex, better defined vessel networks. Conversely, presentation of PEG-IKVAV in combination with PEG-RGDS induced vessels of smaller diameter, suggesting that the type of peptide presented in vivo plays a critical role in determining vessel morphology.

\section{Discussion}

Survival of engineered tissues depends heavily upon the formation of a microvasculature network to achieve sufficient perfusion. Delay in the development of vessels throughout engineered constructs can cause local oxygen and nutrients to deplete, contributing to the death of transplanted cells and failure of the implant. Our current study exploiting immobilized laminin-derived peptides to boost cell-cell interactions shows the efficacy of an alternative approach for forming long-lasting tubule structures. Specifically, immobilized RGDS, YIGSR, and IKVAV, which are known to influence cell migration and invasion, have been used to induce tubule formation in biofunctional hydrogels. In 3D degradable scaffolds, PEG-YIGSR and PEG-IKVAV alone and in combination with PEG-RGDS induced HUVEC tubule formation as early as 7 days, with some groups showing persistent tubules up to 4 weeks. This result is desirable because one of the main aims in tissue engineering is to rapidly form stable vessels that can promote the survival of implanted cells. In comparison with all other treatment groups, cells exposed to PEG-YIGSR in combination with PEG-RGDS arranged in more stable tubule networks with a significant increase in total tubule length and average diameter over a 4-week period. For example, at 28 days, tubules in hydrogels with PEG-YIGSR and PEG-RGDS were $86 \%$ greater in length than those with only PEG-RGDS. The biological effects of YIGSR are mediated by its binding to a $67-\mathrm{kDa}$ laminin receptor. ${ }^{18,28}$ The $67-\mathrm{kDa}$ laminin receptor mediates cell attachment and spreading. Increased expression of the receptor correlates with cell proliferation and migration. ${ }^{28}$ Endothelial cells have been reported to express high levels of $69-\mathrm{kDa}$ laminin-binding receptor that is localized with actin filaments. ${ }^{29}$ The carboxyl terminal of $67-\mathrm{kDa}$ laminin receptor binds to the peptide YIGSR which induces down regulation of mitogen-activated protein kinase phosphatase-1 (MKP-1) expression and dephosphorylation of mitogenactivated protein kinase (MAPK) ${ }^{28}$ Higher MAPK activity induced by the $67-\mathrm{kDa}$ laminin receptor has been implicated previously to correlate with expression of MMPs and to enhance vessel invasion, growth, and angiogenesis. ${ }^{30}$

The trend of increased vessel growth in the presence of YIGSR correlates well with previous in vivo and in vitro studies that have shown the peptide promotes attachment, proliferation, migration, and spreading of endothelial cells. ${ }^{17-19,21}$ In a recent study, endothelial cells seeded in polyurethane urea-modified PEG scaffolds containing YIGSR showed higher levels of cell migration and collagen production. ${ }^{21}$ Other studies have shown that when RGDS and YIGSR sequences were covalently attached to amino phase glass, endothelial cell attachment and spreading were enhanced significantly. ${ }^{31}$ Additionally, though RGDS has been shown to promote cell binding, YIGSR enhances cell-to-cell interactions. ${ }^{18,19}$ Results from the current study suggest that if RGDS and YIGSR are presented simultaneously from the commencement of complex cell interactions, their signaling is synergistic and promotes formation of mature, stable vessels. In fact, vessel density was significantly higher in hydrogels with both YIGSR and RGDS then hydrogels with YIGSR alone. The possibility of a synergistic effect is further supported by the fact that collagen IV production for the PEG-RGDS/PEG-YIGSR combination was significantly greater for the duration of the 4 weeks as compared with any of the other experimental groups. The in vivo results corroborate this finding with a significantly greater vessel density and more extensive branching for the PEG-YIGSR/ PEG-RGDS treatment as compared to PEG-YIGSR alone. After only 7 days in vivo, the average blood vessel density under combined PEG-YIGSR and PEG-RGDS conditions was approximately twice that of hydrogels with only PEG-RGDS or only PEG-YIGSR. This suggests that the presence of the RGDS peptide further enables YIGSR to initiate formation of complex, defined vasculature. Previous studies have reflected signs of a synergistic relationship between these two peptides. $^{31,32}$ In a study of bovine artery cell adhesion, cells exposed to YIGSR and RGDS sequences in combination adhered more readily to amino phase glass. ${ }^{31}$ The synergistic effect was also observed in modified fluorinated ethylene propylene films, which displayed greater neural cell aggregation and anchorage in the presence of the two peptides. ${ }^{32}$ These combination effects on cell adhesion, aggregation, and motility increase the likelihood that such a relationship may hold true for tubule formation in angiogenesis as well.

Hydrogels modified with laminin-derived peptides and implanted in the mouse cornea induced vessel formation, with a particularly heightened response when the materials contained a combination of YIGSR and RGDS. These in vivo results seem to indicate that the morphology of induced vessels can be tuned based on the types of biomimetic peptides presented. In other words, tissues requiring tortuous vessels with large diameters and a greater number of branch points might require a different cocktail of peptides than those with more uniform, small diameter vessels. In the current study, fractal dimension was quantified in order to assess vessel complexity and definition, while lacunarity was measured to examine cell distribution uniformity within the vasculature. $^{27}$ Interestingly, the addition of RGDS to IKVAV or YIGSR causes the fractal dimension and the lacunarity parameter to increase as compared with IKVAV or YIGSR alone. This suggests that with the addition of specific peptides the complexity and distribution of tubules can be altered. Results also show that the response to a YIGSRRGDS combination includes vessels of greater diameters than IKVAV-RGDS. Further, tubules formed in gels with YIGSR or IKVAV co-immobilized with RGDS have a larger tubule diameter in vitro than gels with YIGSR or IKVAV alone. This suggests that specific combination of these peptides results in different vessel morphologies. Hydrogels with IKVAV or RGDS immobilized separately show no change in total tubule length or diameter over 4 weeks in vitro. Similar findings were illustrated when combinations of cell adhesion peptides derived from laminin (YIGSR and PDSGR) and fibronectin (PHSRN and RGDS) were covalently attached to a PDMS substrate to facilitate the adhesion of corneal epithelial cells. ${ }^{33}$ Multiple peptides on the substrate 
resulted in greater adhesion than modification with the individual peptides.

The mechanism by which these laminin-derived peptides stabilize tubule formation may be related to production of basement membrane proteins, including laminin and collagen IV, because the ECM layer is necessary for tissue development and vessel stabilization. ${ }^{32,34}$ In the current work, the accumulations of both laminin and collagen IV were shown to depend on the type and combination of peptides presented, and laminin deposition, in particular, varied significantly with time in culture. Previous studies suggest that the varied composition and structural organization of the ECM during vessel development may have important effects on endothelial cell behavior and tubule morphogenesis. ${ }^{32,34}$ In this process, the maturation of vessels is accompanied by an increase in laminin, 6,35 and collagen IV influences the structural integrity of the basement membrane. ${ }^{36,37}$ Endothelial cell binding to laminin and collagen IV is mediated by integrins and influences cell migration and proliferation. ${ }^{6,35-37}$ Cell binding to both proteins is regulated by the $\alpha_{\mathrm{v}} \beta_{3}$ integrin, which is the same integrin that was shown to induce endothelial cell adhesion in response to immobilized peptide fragments such as IKVAV and YIGSR. ${ }^{38}$ As such, inhibition of $\alpha_{v} \beta_{3}$ integrins has been shown to prevent deposition of ECM proteins. ${ }^{39,40}$ Furthermore, several findings suggest that laminin fragments such as IKVAV and YIGSR can induce greater susceptibility to cell-triggered proteolysis, which permits cell invasion and subsequent ECM matrix remodeling, both of which are necessary for tubule formation. ${ }^{41,42}$

\section{Conclusion}

In this study, we have shown that cellular response can be augmented by introducing short laminin-derived peptide sequences to synthetic scaffolds. In particular, combinations of these biomimetic sequences, which are known to regulate the adhesion, migration, and proliferation of endothelial cells, instigated the formation of stable tubules with well-developed ECM in vitro and in vivo. ${ }^{20,43}$ The covalently immobilized cell adhesion ligands PEG-RGDS, PEG-IKVAV, and PEG-YIGSR have differing effects on tubule formation when presented to endothelial and pericyte precursor cells individually or in combinations. Presentation of a PEG-RGDS/PEG-YIGSR combination induced greater tubule length and diameter in vitro, while resulting in greater vessel density and branching in vivo. In the in vitro experiment, the amounts of collagen IV and laminin, which are ECM proteins involved in vessel stabilization, increased with time from 7 to 28 days and were also found to be dependent on the type of peptides present. Finally in a mouse cornea angiogenesis model, hydrogels incorporating a combination of PEG-YIGSR and PEG-RGDS resulted in vessels with a statistically significant increase in key morphological parameters, highlighting the ability of this formulation to encourage microvascular network formation in tissue engineered constructs. Overall, the results of this study indicate that laminin-derived peptides localized in a polymer matrix can enhance the formation of blood vessels within the construct. Enhancing vascularization in synthetic scaffolds can promote survival of transplanted cells and ultimately compensate for the loss of function in tissues or organs.

\section{Acknowledgments}

This work was supported by NIH R01 HL097520 to MED and JLW and AHA PRE41900219. The authors would like to specially recognize Dr. Melissa McHale (Rice University) for assistance in editing the paper, Aakash H. Keswani (Rice University) for conducting cell culture and data analysis, and to Dr. Tegy Vadakkan (Baylor College of Medicine) for assistance with the analysis of vessel morphology. The authors also gratefully acknowledge support from the Optical Imaging and Vital Microscopy core at Baylor College of Medicine.

\section{Disclosure Statement}

No competing financial interests exist.

\section{References}

1. Atala A, Bauer SB, Soker S, Yoo JJ, Retik AB. Tissue-engineered autologous bladders for patients needing cystoplasty. Lancet. 2006;367:1241-1246.

2. Jain RK, Au P, Tam J, Duda DG, Fukumura D. Engineering vascularized tissue. Nat Biotechnol. 2005;23:821-823.

3. Francis ME, Uriel S, Brey EM. Endothelial cell-matrix interactions in neovascularization. Tissue Eng Part B Rev. 2008;14: 19-32.

4. Nicosia RF, Madri JA. The microvascular extracellular matrix: developmental changes during angiogenesis in the aortic ring-plasma clot model. Am J Pathol. 1987;128:78-90.

5. Miner JH. Laminins and their roles in mammals. Microsc Res Tech. 2008;71:349-356.

6. Hamill KJ, Kligys K, Hopkinson SB, Jones JCR. Laminin deposition in the extracellular matrix: a complex picture emerges. J Cell Sci. 2009;122:4409-4417.

7. Barczyk M, Carracedo S, Gullberg D. Integrins. Cell Tissue Res. 2010;339:269-280.

8. Sreejalekshmi KG, Nair PD. Biomimeticity in tissue engineering scaffolds through synthetic peptide modifications-altering chemistry for enhanced biological response. J Biomed Mater Res. 2011;96:477-491.

9. Ranieri JP, Bellamkonda R, Bekos EJ, Vargo TG, Gardella JA, Aebischer P. Neuronal cell attachment to fluorinated ethylene propylene films with covalently immobilized laminin oligopeptides YIGSR and IKVAV. J Biomed Mater Res. 1995;29:779-785.

10. Miller JS, Shen CJ, Legant WR, Baranski JD, Blakely BL, Chen CS. Bioactive hydrogels made from step-growth derived PEG-peptide macromers. Biomaterials. 2010;31:3736-3743.

11. Moon JJ, Saik JE, Poché RA, et al. Biomimetic hydrogels with pro-angiogenic properties. Biomaterials. 2010;31; 3840-3847.

12. Massia SP, Hubbell JA. Human endothelial cell interactions with surface-coupled adhesion peptides on a nonadhesive glass substrate and two polymeric biomaterials. J Biomed Mater Res. 1991;25:223-242.

13. Massia SP, Hubbell JA. An RGD spacing of 440nm is sufficient for integrin alpha $\mathrm{V}$ beta 3-mediated fibroblast spreading and 140 nm for focal contact and stress fiber formation. J Cell Biol. 1991;114:1089-1100.

14. Grant DS, Kinsella JL, Fridman R, et al. Interaction of endothelial cells with a laminin A chain peptide (SIKVAV) in vitro and induction of angiogenic behavior in vivo. J Cell Physiol. 1992;153:614-625.

15. Nakamura M, Mie M, Mihara H, Nakamura M, Kobatake E. Construction of multi-functional extracellular matrix 
proteins that promote tube formation of endothelial cells. Biomaterials. 2008;29:2977-2986.

16. Grant DS, Zukowska Z. Revascularization of ischemic tissues with SIKVAV and neuropeptide Y (NPY). Adv Exp Med Biol. 2000;476:139-154.

17. Dee KC, Andersen TT, Bizios R. Cell function on substrates containing immobilized bioactive peptides. In Materials Research Society Symposium Proceedings. 1993;331:115-119.

18. Massia SP, Rao SS, Hubbell JA. Covalently immobilized laminin peptide Tyr-Ile-Gly-Ser-Arg (YIGSR) supports cell spreading and co-localization of the 67-kilodalton laminin receptor with alpha-actinin and vinculin. J Biol Chem. 1993; 268:8053-8059.

19. Grant DS, Tashiro K, Segui-Real B, Yamada Y, Martin GR, Kleinman HK. Two different laminin domains mediate the differentiation of human endothelial cells into capillary-like structures in vitro. Cell. 1989;58:933-943.

20. Jun H, West JL. Development of a YIGSR peptide-modified polyurethaneurea to enhance endothelialization. J Biomater Sci Polym Ed. 2004;15:73-94.

21. Jun $H$, West JL. Modification of polyurethaneurea with PEG and YIGSR peptide to enhance endothelialization without platelet adhesion. J Biomed Mater Res B Appl Biomater. 2005;72:131-139.

22. West JL, Hubbell JA. Polymeric biomaterials with degradation sites for proteases involved in cell migration. Macromolecules. 1999;32:241-244.

23. Khoo CP, Micklem K, Watt SM. A comparison of methods for quantifying angiogenesis in the Matrigel assay in vitro. Tissue Eng Part C Methods. 2011;17;895-906.

24. Adighibe $\mathrm{O}$, Micklem K, Campo L, et al. Is nonangiogenesis a novel pathway for cancer progression? A study using 3dimensional tumour reconstructions. Br J Cancer. 2006;94: 1176-1179.

25. Poché RA, Larina IV, Scott ML, Saik JE, West JL, Dickinson ME. The Flk1-myr::mCherry mouse as a useful reporter to characterize multiple aspects of ocular blood vessel development and disease. Dev Dyn. 2009;238:2318-2326.

26. Larina IV, Shen W, Kelly OG, Hadjantonakis AK, Baron MH, Dickinson ME. A membrane associated mCherry fluorescent reporter line for studying vascular remodeling and cardiac function during murine embryonic development. Ana Rec. 2009;292:333-341.

27. Gould DJ, Vadakkan TJ, Poché RA, Dickinson ME. Multifractal and lacunarity analysis of microvascular morphology and remodeling. Microcirculation. 2011;18:136-151.

28. Givant-Horwitz V, Davidson B, Reich R. Laminin-induced signaling in tumor cells. Cancer Lett. 2005;223:1-10.

29. Yannariello-Brown J, Wewer U, Liotta L, Madri JA. Distribution of a $69-\mathrm{kD}$ laminin-binding protein in aortic and microvascular endothelial cells: modulation during cell attachment, spreading, and migration. J Cell Biol. 1988;106:1773-1786.

30. Davidson B, Givant-Horwitz V, Lazarovici P, et al. Matrix metalloproteinases (MMP), EMMPRIN (extracellular matrix metalloproteinase inducer) and mitogen-activated protein kinases (MAPK): co-expression in metastatic serous ovarian carcinoma. Clin Exp Metastasis. 2003;20:621-631.

31. Kouvroukoglou S, Dee KC, Bizios R, McIntire LV, Zygourakis K. Endothelial cell migration on surfaces modified with immobilized adhesive peptides. Biomaterials. 2000;21:17251733.

32. Davis GE, Senger DR. Endothelial extracellular matrix: biosynthesis, remodeling, and functions during vascular morphogenesis and neovessel stabilization. Circ Res. 2005;97: 1093-1107.

33. Merrett K, Griffith CM, Deslandes Y, Pleizier G, Dubé MA, Sheardown $H$. Interactions of corneal cells with transforming growth factor beta 2-modified poly dimethyl siloxane surfaces. J Biomed Mater Res A. 2003;67:981-993.

34. Davis GE, Senger DR. Extracellular matrix mediates a molecular balance between vascular morphogenesis and regression. Curr Opin Hematol. 2008;15:197-203.

35. Risau W, Lemmon V. Changes in the vascular extracellular matrix during embryonic vasculogenesis and angiogenesis. Dev Biol. 1988;125:441-450.

36. Kruegel J, Miosge N. Basement membrane components are key players in specialized extracellular matrices. Cell Mol Life Sci. 2010;67:2879-2895.

37. Khoshnoodi J, Pedchenko V, Hudson BG. Mammalian collagen IV. Microsc Res Tech. 2008;71:357-370.

38. Hodivala-Dilke KM, Reynolds AR, Reynolds LE. Integrins in angiogenesis: multitalented molecules in a balancing act. Cell Tissue Res. 2003;314:131-144.

39. Martinez-Lemus LA, Hill MA, Meininger GA. The plastic nature of the vascular wall: a continuum of remodeling events contributing to control of arteriolar diameter and structure. Physiology. 2009;24:45-57.

40. Iruela-Arispe ML, Davis GE. Cellular and molecular mechanisms of vascular lumen formation. Dev Cell. 2009;16:222-231.

41. Ghosh S, Stack MS. Proteolytic modification of laminins: functional consequences. Microsc Res Tech. 2000;51:238-246.

42. Kuratomi $Y$, Nomizu M, Tanaka K, et al. Laminin gamma 1 chain peptide, C-16 (KAFDITYVRLKF), promotes migration, MMP-9 secretion, and pulmonary metastasis of B16 - F10 mouse melanoma cells. Br J Cancer. 2002;16:1169-1173.

43. Lin X, Takahashi K, Liu Y, Zamora PO. Enhancement of cell attachment and tissue integration by a IKVAV containing multidomain peptide. Biochim Biophys Acta. 2006;1760:1403-1410.

Address correspondence to: Jennifer L. West, PhD

Department of Biomedical Engineering Duke University

Room 3395, CIEMAS Building

Duke University, West Campus

Durham, NC 27708

E-mail: jennifer.1.west@duke.edu 